\title{
Recent Advances in Burkholderia mallei and $B$. pseudomallei Research
}

\author{
Christopher L. Hatcher $^{1}$ • Laura A. Muruato ${ }^{2}$ • Alfredo G. Torres Med,3 $^{1,2}$
}

Published online: 25 March 2015

(C) Springer International Publishing AG 2015

\begin{abstract}
Burkholderia mallei and Burkholderia pseudomallei are Gram-negative organisms, which are etiological agents of glanders and melioidosis, respectively. Although only $B$. pseudomallei is responsible for a significant number of human cases, both organisms are classified as Tier 1 Select Agents and their diseases lack effective diagnosis and treatment. Despite a recent resurgence in research pertaining to these organisms, there are still a number of knowledge gaps. This article summarizes the latest research progress in the fields of $B$. mallei and B. pseudomallei pathogenesis, vaccines, and diagnostics.
\end{abstract}

Keywords Glanders · Melioidosis - B. mallei . B. pseudomallei $\cdot$ Vaccines · Diagnostics $\cdot$ Pathogenesis

\section{Introduction}

Burkholderia mallei and Burkholderia pseudomallei are Gram-negative bacilli which share over $99 \%$ genetic homology. B. mallei is an obligate mammalian pathogen and the

This article is part of the Topical Collection on Bacterial Tropical Medicine

Christopher L. Hatcher and Laura A. Muruato contributed equally to this work.

Alfredo G. Torres holds a PhD degree, University of Texas Medical Branch.

\section{Alfredo G. Torres}

altorres@utmb.edu

1 Department of Microbiology and Immunology, University of Texas Medical Branch, Galveston, TX 77555, USA

2 Institute for Translational Sciences, University of Texas Medical Branch, Galveston, TX, USA

3 Department of Pathology, University of Texas Medical Branch, Galveston, TX, USA causative agent of glanders, a disease that primarily affects solipeds (horses, mules, and donkeys). Glanders is endemic to parts of Africa, Asia, Middle East, Central, and South America [1]. Naturally acquired human cases typically occur among persons with prolonged contact with solipeds, and the major routes of transmission are cutaneous and respiratory. Clinical manifestations are route dependent and include $a b-$ scesses, fever, pneumonia, dissemination to the liver and spleen causing necrotizing abscesses, and bacteremia [2]. B. pseudomallei is a saprophyte of wet soils and are endemic to tropical and subtropical areas around the world [3]. This organism can infect various mammals and is the causative agent of melioidosis in humans. Melioidosis symptoms are similar to those described for glanders, but in addition to cutaneous and respiratory routes of infection, melioidosis is also acquired by the gastrointestinal route [4]. B. pseudomallei is a leading cause of sepsis in Northern Australia [5] and bacterial pneumonia in Thailand [6]. Both glanders and melioidosis have high mortality rates (up to $50 \%$ ), even when aggressive antimicrobial therapy is employed [7]. Additionally, both species are classified as Tier 1 Select Agents by the US Federal Select Agent Program because of their biothreat potential and intrinsic characteristics, including low infectious doses, high morbidity and mortality rates, multi-drug antibiotic resistance, and amenability to aerosolize [8]. Although $B$. mallei and B. pseudomallei research has seen significant advancements, there are still large gaps in the understanding of these pathogens and their interactions with the host. This report highlights recent progress made regarding in the areas of pathogenesis, vaccinology, and diagnostics (Fig. 1).

\section{Pathogenesis}

B. mallei and B. pseudomallei possess multiple secretion systems that facilitate invasion and replication within host cells. These systems play a major role in pathogen virulence, and provide novel targets for the development of vaccines and therapeutics. 


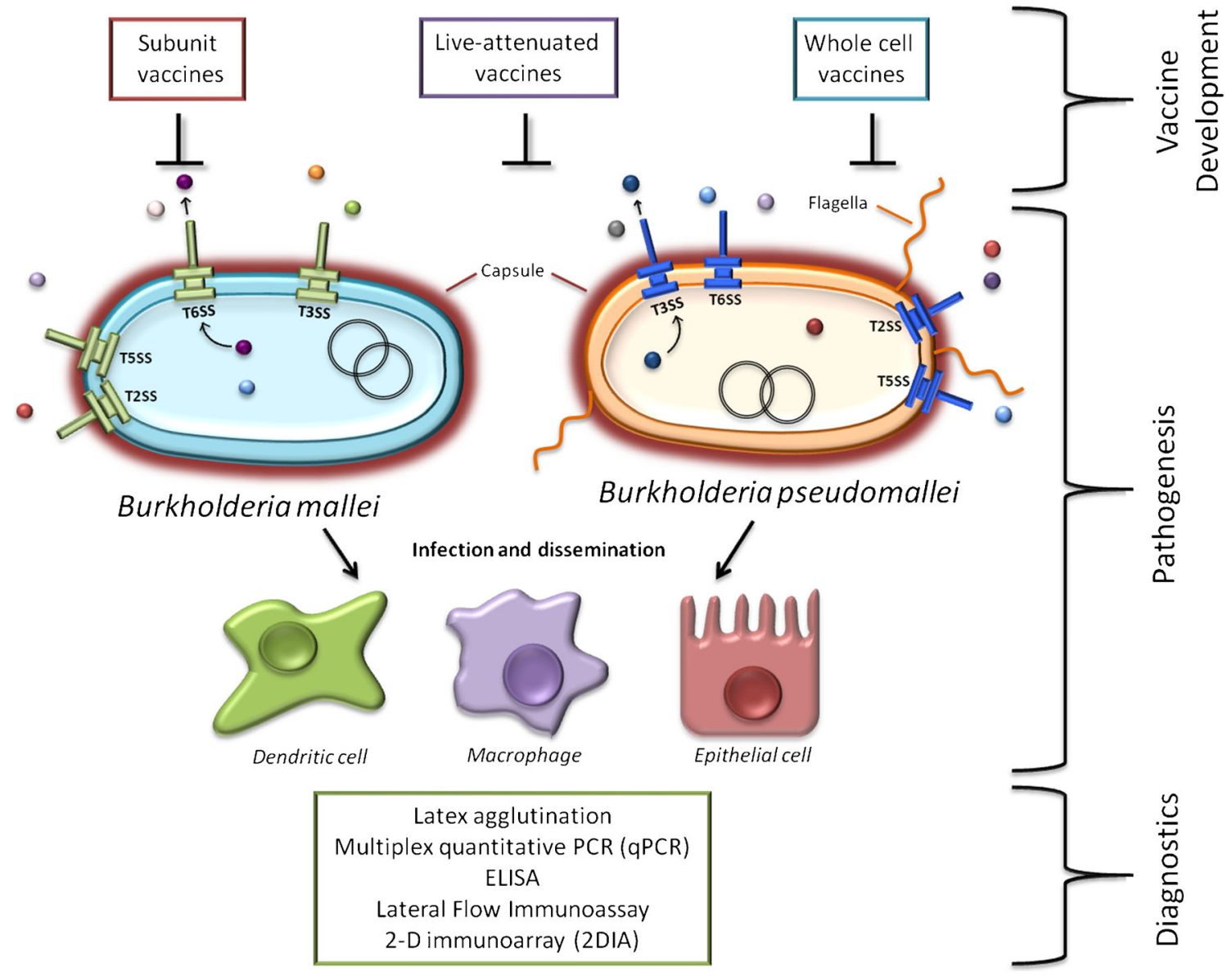

Fig. 1 Graphic representation of Burkholderia mallei and B. pseudomallei pathogenesis, vaccine development, and emerging diagnostic tools. T2SS, T3SS, T5SS, and T6SS refer to secretion systems that contribute to pathogenesis in both organisms

\section{Type 2 Secretion System}

Until recently, the type 2 secretion system (T2SS) has been poorly characterized in B. pseudomallei. Burtnick et al. carried out one of the first analyses of the T2SS proteome [9]. The T2SS relies on the ATPase GspE and the outer membrane channel GspD for function. Construction and analysis of inframe deletion mutants of $g s p E$ and $g s p D$ allowed the identification of $\sim 50$ proteins secreted by the T2SS. Among these were numerous hydrolytic enzymes and the deubiquitinase TssM, which has previously been shown to be immunogenic via reaction with human melioidosis patient sera [10]. Unexpectedly, the current study revealed that deletion of the T2SS slightly increases mutant virulence, as demonstrated by a decreased $\mathrm{LD}_{50}$ and time to death in a BALB/c mouse model [9]. The mechanism behind this increase in virulence remains to be elucidated.

\section{Type 3 Secretion System}

A functional type 3 secretion system (T3SS) is essential for B. pseudomallei escape from the phagolysosome following invasion. Virulence factors such as BopA, BipB, and BipD have previously been reported to play a pivotal role in phagosomal escape, multi-nucleated giant cell (MNGC) formation, and intracellular replication. Kang et al. have characterized another Burkholderia invasion protein, BipC, demonstrating its role in virulence [11]. A bipC mutant was constructed from B. pseudomallei K96243 and evaluated in human lung epithelial cells. The mutant displayed decreased invasion, adherence, and intracellular survival in vitro. Additionally, the bipC mutant exhibited a delay in actin-based motility and phagosomal escape. Similar findings were observed in vivo, confirming the decreased virulence of the mutant by increased survival and healthier overall appearance of i.p. challenged BALB/c mice. Therefore, BipC appears to play an essential role in the overall pathogenicity and virulence of B. pseudomallei.

\section{Type 5 Secretion System}

Autotransporters comprise the largest family of proteins secreted by the type 5 secretion system (T5SS). The functions of autotransporters are diverse, including adhesion, invasion, 
serum resistance, intracellular survival, and biofilm formation. Among the well-described Burkholderia autotransporters are $\mathrm{BimA}$ and $\mathrm{BoaA} / \mathrm{BoaB}$, which function in actin polymerization and adhesion, respectively. Further characterization of $\mathrm{BpaC}, \mathrm{BcaA}$, and $\mathrm{BcaB}$ autotransporters has recently been reported. A study by Lafontaine et al. demonstrated that $\mathrm{BpaC}$ plays an important role in adhesion [12]. In vitro analysis of bpaC-transformed Escherichia coli revealed increased adhesion to human lung, laryngeal, and bronchial epithelial cells. Similar studies were conducted with $b p a C$ mutants of $B$. mallei ATCC 23344 and B. pseudomallei DD503. Interestingly, while the $B$. mallei mutant showed decreased adhesion in all three cell types, the $B$. pseudomallei mutant portrayed decreased adhesion only to the bronchial epithelium. However, changes in adhesion did not appear to affect virulence of the mutants in vivo, as indicated by equivalent $\mathrm{LD}_{50}$ values and bacterial burden in an aerosol mouse model of infection [12].

Another study by Campos et al. demonstrated that B. pseudomallei strain $340 \mathrm{bcaA}$ and $b c a B$ mutants displayed impaired invasion and decreased plaque formation in lung epithelial cells [13]. Furthermore, a BALB/c i.n. model of acute infection showed significantly fewer bacteria recovered from the spleen of mice infected with $\Delta b c a A$, suggesting that BcaA may play a role in dissemination to the spleen.

\section{Type 6 Secretion System}

Type 6 secretion system (T6SS) is necessary for MNGC formation and plays an important role in intracellular growth and actin-based motility. Hemolysin co-regulated protein-1 (Hcp1), located within T6SS cluster-1, and it has been well characterized for its role in MNGC formation and intracellular survival. By altering the divalent ion concentrations in minimal media, it was shown that the addition of iron and zinc negatively regulates in vitro expression of T6SS cluster-1 genes. This negative regulation was demonstrated by significant reduction in $h c p 1$ expression in B. mallei SR1A and B. pseudomallei K96243 and 1026b [14••]. By optimizing growth conditions, the highest expression of Hcp1 by both Burkholderia was observed in minimal salts media lacking iron and zinc and supplemented with $0.4 \%$ glucose and $0.5 \%$ bacto-casamino acids. Furthermore, it was determined that VirG positively regulates expression of both Burkholderia T6SS-1 systems, as demonstrated by negligible production of Hcp-1 when a vir $G$ mutant was analyzed.

Hopf et al. performed further characterization of the T6SS1 in a study that described the T6SS-1 gene, BPSS1504, and its apparent effect on Hcp1 secretion. A BPSS1504 mutant showed no difference in $h c p 1$ mRNA levels; however, secreted Hcp1 protein was unable to be detected. In addition to abrogated Hcp 1 secretion, the mutant exhibited decreased virulence in vivo and in vitro. These findings suggest that
BPSS1504 may play a role in the functionality of the T6SS1 apparatus [15].

A member of the $v g r$ family, VgrG5, has been reported to play a role in the virulence of B. pseudomallei. VgrG5 was thoroughly characterized by Toesca et al. and Schwarz et al. $[16,17]$. Both confirmed that VgrG5 functions in MNGC in vitro formation and reported that the $\mathrm{C}$-terminal domain (CTD) of VgrG5 is essential for its function. Additionally, both groups constructed Burkholderia thailandensis vgrG5 mutants for evaluation in vitro and reported that they were unable to induce membrane fusion. In order to further characterize the role of CTD, Schwarz et al. performed in vivo studies in a C57BL/6 mouse model via aerosol challenge of B. thailandensis CTD mutant. All mice challenged with $\Delta$ CTD survived $>14$ days post-infection, whereas mice infected with wild-type (WT) succumbed to infection between days 2-4. Additionally, Toesca et al. demonstrated high conservation of VgrG5 between Burkholderia species. By crosscomplementing a $B$. pseudomallei $v g r G 5$ mutant with $v g r G 5$ alleles from B. mallei ATCC 22344, B. pseudomallei MSHR668, B. oklahomensis C6786, and B. thailandensis E264, this group was able to restore membrane fusion capability and MNGC formation in vitro.

\section{Surface Polysaccharides}

Capsular polysaccharide (CPS) and lipopolysaccharide (LPS) are the best well-described surface antigens expressed by both Burkholderia species. Because passive immunization with CPS and LPS has previously been shown to provide partial protection from infection, current vaccine studies focus on incorporating LPS or CPS for enhanced immunity. Although B. pseudomallei LPS has been considered weakly antigenic in experimental models, a recent study by Chantratita et al. suggests that LPS plays an important role in stimulating the human innate immune response to B. pseudomallei infection [18]. Stimulation of human monocytes with heat-killed B. pseudomallei 1026b or K96243 in the presence of LPS antagonist polymyxin B or TLR4 neutralizing antibody showed dramatic decrease in TNF- $\alpha$ production. Additionally, stimulation of whole blood with purified B. pseudomallei LPS generated a greater cytokine response than $E$. coli LPS. Taken together, these data suggest an important role for $B$. pseudomallei LPS in initiating innate cytokine production [18].

\section{Quorum Sensing}

The role of quorum sensing (QS) in the pathogenesis of $B$. mallei remains controversial. Ulrich et al. reported that B. mallei QS mutants showed severe attenuation both in vitro and in vivo [19]. However, a recent report by Majerczyk et al. suggests that QS is not essential for acute 
infection [20]. In order to more fully elucidate the QS role in B. mallei infection, the horse-passaged strain GB8 was used to construct unmarked, in-frame deletions of the homoserine lactone (HSL) synthases genes bmaIl and bmal3. Additionally, the B. mallei strain ATCC 23344 was used to generate an acylhomeoserine lactone (AHL) lactonase-producing mutant. In vitro experiments were performed using murine macrophage-like RAW 264.7 cells. Intracellular replication of GB8$\Delta b m a I 1, \mathrm{~GB} 8 \Delta b m a I 3$, and GB8 $\Delta b m a I 1 / \Delta b m a I 3$ were indistinguishable from WT parent strain. Similar results were observed in a BALB/c mouse model, with comparable survival times seen between WT and mutant-challenged mice. Additionally, the B. mallei AHL-lactonase-producing strain CM4 demonstrated WT virulence in vivo. In order to assess the functionality of the bmaIl promoter, $\mathrm{BALB} / \mathrm{c}$ mice were infected with the luminescent fusion strain CM4. High fluorescence was visible in the lungs at disease onset, indicating that the bmall gene is active during infection. These results suggest that QS is not required for acute $B$. mallei infection, but its role in chronic infection has not yet been characterized.

Likewise, the role of quorum sensing in closely related B. pseudomallei is poorly understood. Recently, reports suggest that QS may play a role in MNGC formation by B. pseudomallei. Horton et al. developed AHL-synthase mutants of the bpsI1, bpsI2, and bpsI3 genes in strain MSHR520 [21]. Interestingly, the triple mutant $\Delta b p s I 123$ showed significant increase in MNGC formation compared to WT. However, no significant difference was seen in the intracellular survival or replication of the mutants. Additionally, comparable virulence was observed between $\Delta$ bpsI123 and WT, as determined by CFU counts of blood, spleen, lungs, and liver tissues of challenged $\mathrm{BALB} / \mathrm{c}$ mice.

\section{Host-Pathogen Interaction}

During infection, the release of stress hormones functions to regulate many physiological responses including inflammation and metabolic activity. Intarak et al. describe the role of epinephrine on B. pseudomallei pathogenicity in vitro [22]. Epinephrine concentration, ranging from 50 to $200 \mu \mathrm{M}$, was shown to have a positive effect on bacterial growth, which was significantly increased over untreated cultures. Because catecholamines, like epinephrine, are believed to function in the release of free iron, the relationship of iron to bacterial growth was analyzed in vitro. The addition of $\mathrm{FeCl}_{3}$ was shown to increase $B$. pseudomallei growth, whereas administering the iron-chelating agent deferoxamine (DFO), in the presence of epinephrine, dramatically decreased growth. Additionally, treatment with $50 \mu \mathrm{M}$ epinephrine resulted in increased radial motility and expression of flagellar genes, including fliC, fliA, flhD, and motA. These results suggest that epinephrine contributes to increased growth, gene expression, and motility in vitro.
Williams et al. showed that dendritic cells facilitate dissemination of $B$. pseudomallei NCTC 13179 during acute infection [23•]. B. pseudomallei was shown to induce maturation of bone marrow-derived dendritic cells (DCs) and stimulate in vitro migration. Importantly, DCs were shown to retain intracellular $B$. pseudomallei during migration, indicating a potential for trafficking. In both in vitro and in vivo studies, B. pseudomallei infection resulted in a more potent stimulation of DCs than LPS, as indicated by increased expression of maturation markers and increased migration. The ability of DCs to facilitate spread of $B$. pseudomallei was confirmed in vivo in a C57BL/6 mouse model. This study confirms that DCs are able to traffic $B$. pseudomallei from the site of cutaneous infection to the spleen and lungs and thereby, facilitating bacterial dissemination.

Mulye et al. also demonstrated the importance of the innate immune system by elucidating the role of serum opsonins in the uptake and killing of $B$. pseudomallei by neutrophils and macrophages [24]. Increased complement deposition was shown to occur on the $B$. pseudomallei acapsular mutant SZ210. Additionally, B. pseudomallei, its acapsular mutant, and $B$. thailandensis all showed resistance to direct killing by complement, even when incubated in high concentrations of serum. While serum opsonization resulted in increased uptake of B. pseudomallei by both neutrophils and macrophages, intracellular killing by macrophages remained unaffected. In contrast, decreased intracellular survival within neutrophils was observed when bacteria were opsonized with $\geq 5 \%$ serum. These data suggest that opsonization functions to increase uptake and may play a role in intracellular killing by neutrophils.

\section{Vaccine Development}

Based on the high incidence of melioidosis in endemic areas, the potential for re-emergence of glanders, and both diseases potential for bioterrorist use, development of effective vaccines against these diseases is an ideal approach to prevent infection. Currently, no vaccine candidates have been selected for glanders or melioidosis human or veterinary prevention trials. Numerous vaccine reviews and studies suggest that both cellular and humoral immune responses are required for complete protection against these pathogens [25-28]. Therefore, a summary of the recently evaluated $B$. mallei and $B$. pseudomallei vaccine candidates is described.

\section{Live Attenuated Vaccines}

Scott et al. looked at the ability of B. thailandensis E555, a non-pathogenic strain producing $B$. pseudomallei-like mannoheptose capsule, to protect against melioidosis. The study demonstrated complete bacterial clearance in the lungs, liver, 
and spleen by day 3 in BALB/c mice vaccinated prior to challenge with $B$. pseudomallei K96243. Sera from BALB/c mice receiving either $B$. thailandensis or a non-encapsulated control strain showed that animals exposed to E555 produced higher $B$. pseudomallei-specific IgG. Finally, mice immunized with E555 prior to challenge with B. pseudomallei K96243 exhibited increased survival rates over mice vaccinated with non-encapsulated control [29].

Mott et al. constructed an unmarked in-frame deletion in the B. mallei ATCC 23344 tonB gene (BMAA1801), creating an iron transport-deficient strain. BALB/c mice intranasally immunized with the $\triangle \operatorname{ton} B$ strain at doses of $1.5 \times 10^{5}$ and $1.5 \times 10^{4} \mathrm{CFU}$ and subsequently challenged with WT $B$. mallei had survival rates of 100 and $75 \%$ respectively. A crossprotection assay of mice vaccinated with $\triangle \operatorname{ton} B$ vaccine and challenged with the WT B. pseudomallei K96243 strain demonstrated that mice had a survival rate of $75 \%$ up to 36 days post-infection. Although highly protective against both pathogens, persistence was noted both by the appearance of splenic abscesses and recovery of $B$. mallei $\Delta \operatorname{ton} B$ strain from the spleens of surviving mice. Despite persistence, the ability of the $B$. mallei ton $B$ mutant to provide cross-protection against both $B$. mallei and B. pseudomallei makes it a potential candidate for further vaccine development and optimization [Mott et al., personal communication].

Silva et al. demonstrated that subcutaneous vaccination can also protect against murine melioidosis. A highly attenuated purM mutant of B. pseudomallei strain 1026b (Bp82) was used to immunize $\mathrm{BALB} / \mathrm{c}$ and $\mathrm{C} 57 \mathrm{BL} / 6$ mice prior to $\mathrm{WT}$ challenge. Results indicated that BALB/c and C57BL6 mice vaccinated with the Bp82 showed 100 and $60 \%$ survival, respectively. Post-immunized sera contained a significantly higher level of IgG and IgM in comparison to controls; likewise, significantly higher levels of IL-17 and IFN- $\gamma$ were also detected. Mice deficient in B cells $\left(\mathrm{uMT}^{-/}\right)$or T cells $\left(\mathrm{CD}^{-/-}\right.$ and $\mathrm{CD} 8^{--}$) were analyzed in response to vaccination and challenge, and those lacking B cells exhibited only $50 \%$ survival. Immune sera transferred to naïve mice resulted in $38 \%$ survival. In contrast, mice deficient in $\mathrm{T}$ cells exhibited survival equivalent to that of vaccinated WT. This study identified correlates of immune protection, specifically highlighting the role of humoral immunity in protecting against B. pseudomallei infection [30•].

\section{Killed Vaccines}

Puangperch et al. evaluated the protective effects of i.m. administration of heat-killed (HK) or paraformaldehyde-killed (PK) B. pseudomallei to BALB/c mice prior to challenge with WT B. pseudomallei. The PK treatment provided increased protection against $B$. pseudomallei over HK (60\% survival vs $0 \%$ ) Additionally, it was shown that PK-immunized mice had decreased bacterial counts in their blood and increased
B. pseudomallei-specific IgG and IFN- $\gamma$ serum levels in comparison to HK immunized mice [31].

\section{Subunit Vaccines}

Outer membrane vesicles (OMVs) are non-infectious particles that contain LPS, CPS, and a milieu of membrane and periplasmic proteins. OMVs have been isolated from B. pseudomallei $1026 \mathrm{~b}$ and have previously been demonstrated as an effective vaccine platform, which provides significant protection (60\% survival) against respiratory murine melioidosis [32]. Nieves et al. showed that s.c. immunization with OMVs also provided protection against lethal septic $\mathrm{BALB} / \mathrm{c}$ mouse infection, as demonstrated by increased survival rates compared to unvaccinated controls [33]. However, splenic persistence in surviving animals from both studies indicated that OMV vaccination only provided partial bacterial clearance.

Petersen et al. have demonstrated the safety and immunogenicity of OMV vaccination in rhesus macaque non-human primates (NHP). In this study, NHPs received either three doses of adjuvant alone (control) or three escalating doses of OMVs plus adjuvant. Safety was evident by lack of pathology at the injection site and normal blood chemistry and hematology results in NHPs. Immunogenicity of the OMVs was demonstrated by significant increases in OMV-specific, LPS-specific, and CPS-specific IgG titers in OMV-vaccinated NHPs compared to controls [34•].

Other studies have investigated the use of $B$. mallei and B. pseudomallei glycoconjugate vaccines. Scott et al. evaluated a conjugate vaccine comprised of covalently joined LPS and tetanus toxin $\mathrm{H}_{\mathrm{c}}\left(\mathrm{TetH}_{\mathrm{c}}\right)$ in a melioidosis mouse model. The conjugate vaccine provided partial protection, resulting in slightly increased survival in comparison to only LPS or unconjugated $\mathrm{TetH}_{\mathrm{c}}$ and LPS. Additionally, although there was no significant difference in splenic colonization between the groups, mice receiving the conjugate had significantly higher titers of IgG1 and IgG2a compared to the other groups [35]. Scott et al. also evaluated a series of unconjugated and conjugated $O$-polysaccharide (OPS) and CPS vaccine preparations combined with cationized bovine sheep albumin (cBSA) and LolC. Both conjugate vaccines were more protective than their unconjugated polysaccharide and cBSA counterparts. The conjugate vaccine containing CPS was more protective than the OPS conjugate, with median time to death of 29.5 and 10 days, respectively. The addition of LolC to the CPS conjugate resulted in increased protection, with immunized mice showing $70 \%$ survival in contrast to $50 \%$ survival with the CPS conjugate alone. The incorporation of LolC resulted in lower bacterial counts in the lungs, livers, and spleens [36].

Gregory et al. developed and evaluated the protective capability of gold nanoparticles (AuNPs) conjugated to purified LPS (AuNP-LPS) and covalently linked to recombinant 
carrier proteins (TetHc, Hcp1, or FliC). Different combinations of the AuNPs were delivered i.n. to BALB/c mice prior to a lethal challenge with $B$. mallei ATCC 23344. At a low challenge dose $\left(\sim 2 \mathrm{LD}_{50}\right)$, LPS, AuNP-LPS, and AuNP-LPS provided similar levels of protection; however, at the high challenge dose $\left(6.5 \mathrm{LD}_{50}\right)$, the groups receiving AuNP showed increased protection and had significantly lower splenic bacterial burdens. Furthermore, sera from the protein-coupled nanoglycoconjugate groups had significantly higher LPS-specific $\operatorname{IgG}$ and $\operatorname{IgM}$ levels than controls [37].

Torres et al. also evaluated the protective capacity of AuNP-LPS coupled with FliC (AuNP-LPS-FliC) in a NHP model of respiratory glanders. AuNP-LPS-FliCvaccinated NHPs demonstrated increased survival over the PBS control group, with survival rates of 50 and $33.3 \%$ in the groups, respectively. While bacterial burdens were detected in infected tissues of surviving control animals, no detectable bacteria were present in organs (spleen, liver, and lungs) of vaccinated animals. Serum LPS-specific IgG titers were significantly increased in vaccinated NHPs than in controls. Overall, results showed that AuNP-LPS-FliC vaccine is immunogenic and provides protection against aerosolized glanders infection $[38 \cdot \bullet]$.

\section{Diagnostics}

The current "gold standard" diagnostic tests for melioidosis and glanders have long turnaround times and are prone to misdiagnosis due to low sensitivity and specificity. A definitive glanders or melioidosis diagnosis via routine bacterial culture typically takes approximately 7 days, and the poor sensitivity of this methodology increases the risk of undetected cases. Conventional serological testing of humans in melioidosis endemic areas is unreliable due to high seroprevalence rates. Additionally, diagnostic shortcomings have been identified in the mallein and compliment fixation test (CFT), which are used to identify glanders-positive solipeds. The mallein test has a reported sensitivity of only $75.7 \%$ [39] and the CFT, while more sensitive (97\%), has been implicated in false-positive results [1]. We next summarize novel detection methods that may address these gaps.

\section{Latex Agglutination Test}

Duval et al. evaluated the latex agglutination test on its ability to accurately detect $B$. mallei and $B$. pseudomallei from culture. The detection reagent in this assay is comprised of latex particles coated with $4 \mathrm{~B} 11$ monoclonal antibody to $B$. mallei and $B$. pseudomallei CPS, which, when combined with a single isolated B. mallei or B. pseudomallei colony on a ringed glass slide and rocked briefly, will agglutinate. Using a panel of 110 B. pseudomallei and B. mallei isolates and a panel of 36 clinically relevant and phylogenetically related Burkholderia spp., the assay had sensitivity of $99.1 \%$ and specificity of $97.2 \%[40]$. Despite the requirement of bacteria to be cultured and isolated prior to identification, the accuracy, ease of use, relatively low cost per test, and negligible requirements for additional equipment make it a potentially invaluable diagnostic tool, particularly in resource-poor areas.

\section{Molecular Diagnostics}

Janse et al. developed a single-reaction quadruplex quantitative real-time PCR for the detection and differentiation of B. pseudomallei and B. mallei from both bacterial culture lysates and various patient samples. The assay includes BuMC, a sequence unique to both $B$. mallei and B. pseudomallei; $p s u$, a sequence unique to B. pseudomallei; and $m a u$, a sequence specific to $B$. mallei. Additionally, primer and probe sets are included in the assay to act as an internal DNA extraction control. This assay was validated via a panel of $10 \mathrm{~B}$. mallei clinical isolates, 19 $B$. pseudomallei isolates, 16 negative bacterial controls, positive autopsy tissue samples (lung, liver, spleen, and prostate) from a melioidosis patient, and 10 negative multispecies blood and tissue samples. The assay correctly identified all negative and B. mallei-positive samples. Although the assay detected psu in all 19 B. pseudomallei samples, it failed to detect BuMC in three of the B. pseudomallei isolates. Although no sensitivity or specificity data was provided, the limit of detection of the assay was estimated to be $<1$ genome equivalent per reaction [41].

Hsu et al. developed a 15-plex assay for the identification of 11 pathogens of clinical and biothreat importance including B. mallei and B. pseudomallei. This Luminex-based assay was validated by performing blind testing with bacterial culture colonies of 57 ATCC reference strains and 1 clinical Brucella spp. isolate, and it correctly identified individual pathogens with $100 \%$ accuracy. This method is able to identify all agents present when mixtures of up to six different bacterial DNAs were assayed. The limit of detection for this assay is between 5 and 100 genomic copies for each of the 11 pathogens [42].

\section{Serological Diagnostics}

Singha et al. developed an indirect ELISA method which was able to identify TssB, a highly antigenic protein of the $B$. mallei T6SS. The assay, evaluated with a panel of 49 known glanders-positive and 40 negative equine serum samples, was able to diagnose with $100 \%$ accuracy. Comparison with the complement fixation test on 1811 equine serum samples 
indicated that 4 of the 1811 samples were false positive by ELISA, but none were false negative. The sensitivity and specificity of this TssB indirect ELISA was determined to be 100 and $99.7 \%$, respectively [43].

Houghton et al. developed and evaluated the Active Melioidosis Detect Lateral Flow Immunoassay (AMD-LFI). Using a single colony of $77 \mathrm{~B}$. pseudomallei isolates, 33 B. mallei isolates, and 36 near-neighbor common clinical isolates, the test showed a sensitivity of $98.7 \%(76 / 77)$ for B. pseudomallei and $90.9 \%$ (30/33) for B. mallei, with an overall specificity of $97.2 \%(35 / 36)$. The limit of detection for this test was calculated to be $0.2 \mathrm{ng} / \mathrm{ml}$ of CPS. Finally, the assay was optimized for its use with a variety of direct patient specimens, including serum, pus, urine, sputum, and pleural fluid [44••].

Indirect hemagglutination assay (IHA) is used in Australia as serodiagnostic test with poor sensitivity. Sorenson et al. developed and evaluated a two-dimensional immunoarray (2DIA) designed to detect the three most common Burkholderia LPS types (A, B, and B2). Using a panel of 60 serum samples, they found that 2DIA had a much higher sensitivity than IHA (100 vs. $58.6 \%)$, giving positive results for all melioidosis culture-positive/IHA-negative serums, but a lower specificity $(87.1$ vs. $100 \%$ [ [45].

\section{Conclusion}

Understanding B. mallei and B. pseudomallei pathogenesis has opened the door to the development of more specific diagnostics and therapies. The advancements in the understanding of host-pathogen interaction, in addition to the identification and purification of bacterial virulence factors, have increased the speed and accuracy of diagnostics and identification of immunogenic vaccine targets. However, identification of vaccine candidates that provide long-term and complete protection as well as sterilizing immunity remains an elusive goal requiring further attention.

\begin{abstract}
Acknowledgments The study performed in the laboratory of AGT was supported by NIH/NIAID grant U54 AI057156. The contents are solely the responsibility of the authors and do not necessarily represent the official views of the NIAID or NIH.
\end{abstract}

\section{Compliance with Ethics Guidelines}

Conflict of Interest Christopher L. Hatcher, Laura A. Muruato, and Alfredo G. Torres declare that they have no conflict of interest.

Human and Animal Rights and Informed Consent This article does not contain any studies with human or animal subjects performed by any of the authors.

\section{References}

Papers of particular interest, published recently, have been highlighted as:

- Of importance

- Of major importance

1. Neubauer H, Sprague LD, Zacharia R, Tomaso H, Al Dahouk S, Wernery R, et al. Serodiagnosis of Burkholderia mallei infections in horses: state-of-the-art and perspectives. J Vet Med B Infect Dis Vet Public Health. 2005;52:201-5.

2. Van Zandt KE, Greer MT, Gelhaus HC. Glanders: an overview of infection in humans. Orphanet J Rare Dis. 2013;8:131.

3. Dance DA. Melioidosis as an emerging global problem. Acta Trop. 2000;74:115-9.

4. Cheng AC, Currie BJ. Melioidosis: epidemiology, pathophysiology, and management. Clin Microbiol Rev. 2005;18:383-416.

5. Currie BJ, Fisher DA, Howard DM, Burrow JN, Lo D, SelvaNayagam S, et al. Endemic melioidosis in tropical northern Australia: a 10-year prospective study and review of the literature. Clin Infect Dis. 2000;31:981-6.

6. Chaowagul W, White NJ, Dance DA, Wattanagoon Y, Naigowit P, Davis TM, et al. Melioidosis: a major cause of community-acquired septicemia in northeastern Thailand. J Infect Dis. 1989;159:890-9.

7. Dance DA. Melioidosis: the tip of the iceberg? Clin Microbiol Rev. 1991;4:52-60.

8. HHS and USDA Select Agents and Toxins 7 CFR Part 331, 9 CFR Part 121, 42 CFR Part 73. 2012. http://www.selectagents.gov/ resources/List_of_Select_Agents_and_Toxins_2012-12-4.pdf. Accessed 1 February 2012.

9. Burtnick MN, Brett PJ, DeShazer D. Proteomic analysis of the Burkholderia pseudomallei type II secretome reveals hydrolytic enzymes, novel proteins, and the deubiquitinase TssM. Infect Immun. 2014;82:3214-26.

10. Tan KS, Chen Y, Lim YC, Tan GY, Liu Y, Lim YT, et al. Suppression of host innate immune response by Burkholderia pseudomallei through the virulence factor TssM. J Immunol. 2010;184:5160-71.

11. Kang WT, Vellasamy KM, Chua EG, Vadivelu J. Functional characterizations of effector protein BipC, a type III secretion system protein, in Burkholderia pseudomallei Pathogenesis. J Infect Dis. 2014. [Epub ahead of print Aug 26].

12. Lafontaine ER, Balder R, Michel F, Hogan RJ. Characterization of an autotransporter adhesin protein shared by Burkholderia mallei and Burkholderia pseudomallei. BMC Microbiol. 2014;14:92.

13. Campos CG, Borst L, Cotter PA. Characterization of BcaA, a putative classical autotransporter protein in Burkholderia pseudomallei. Infect Immun. 2013;81:1121-8.

14.• Burtnick MN, Brett PJ. Burkholderia mallei and Burkholderia pseudomallei cluster 1 type VI secretion system gene expression is negatively regulated by iron and zinc. PLoS One. 2013;8:e76767. This study describes the regulatory effect of divalent ions on $B$. mallei and B. pseudomallei T6SS-1 expression in vitro.

15. Hopf V, Gohler A, Eske-Pogodda K, Bast A, Steinmetz I, Breitbach K. BPSS1504, a cluster 1 type VI secretion gene, is involved in intracellular survival and virulence of Burkholderia pseudomallei. Infect Immun. 2014;82:2006-15.

16. Toesca IJ, French CT, Miller JF. The Type VI secretion system spike protein VgrG5 mediates membrane fusion during intercellular spread by pseudomallei group Burkholderia species. Infect Immun. 2014;82:1436-44.

17. Schwarz S, Singh P, Robertson JD, LeRoux M, Skerrett SJ, Goodlett DR, et al. VgrG-5 is a Burkholderia type VI secretion 
system-exported protein required for multinucleated giant cell formation and virulence. Infect Immun. 2014;82:1445-52.

18. Chantratita N, Tandhavanant S, Myers ND, Seal S, Arayawichanont A, Kliangsa-Ad A, et al. Survey of innate immune responses to Burkholderia pseudomallei in human blood identifies a central role for lipopolysaccharide. PLoS One. 2013;8:e81617.

19. Ulrich RL, Deshazer D, Hines HB, Jeddeloh JA. Quorum sensing: a transcriptional regulatory system involved in the pathogenicity of Burkholderia mallei. Infect Immun. 2004;72:6589-96.

20. Majerczyk C, Kinman L, Han T, Bunt R, Greenberg EP. Virulence of Burkholderia mallei quorum-sensing mutants. Infect Immun. 2013;81:1471-8.

21. Horton RE, Grant GD, Matthews B, Batzloff M, Owen SJ, Kyan S, et al. Quorum sensing negatively regulates multinucleate cell formation during intracellular growth of Burkholderia pseudomallei in macrophage-like cells. PLoS One. 2013;8:e63394.

22. Intarak N, Muangsombut V, Vattanaviboon P, Stevens MP, Korbsrisate S. Growth, motility and resistance to oxidative stress of the melioidosis pathogen Burkholderia pseudomallei are enhanced by epinephrine. Pathog Dis. 2014;72:24-31.

23. Williams NL, Morris JL, Rush CM, Ketheesan N. Migration of dendritic cells facilitates systemic dissemination of Burkholderia pseudomallei. Infect Immun. 2014;82:4233-40. This article highlights the ability of dendritic cells to facilitate dissemination of $B$. pseudomallei from the cutaneous site of infection.

24. Mulye M, Bechill MP, Grose W, Ferreira VP, Lafontaine ER, Wooten RM. Delineating the importance of serum opsonins and the bacterial capsule in affecting the uptake and killing of Burkholderia pseudomallei by murine neutrophils and macrophages. PLoS Negl Trop Dis. 2014;8:e2988.

25. Bondi SK, Goldberg JB. Strategies toward vaccines against Burkholderia mallei and Burkholderia pseudomallei. Expert Rev Vaccines. 2008;7:1357-65.

26. Healey GD, Elvin SJ, Morton M, Williamson ED. Humoral and cell-mediated adaptive immune responses are required for protection against Burkholderia pseudomallei challenge and bacterial clearance postinfection. Infect Immun. 2005;73:5945-51.

27. Mott TM, Estes DM, Torres AG. Recent progress in the development of vaccines for glanders and melioidosis, chapter 4. In: Feodorova VA, Motin VL, editors. Vaccines against bacterial biothreat pathogens. Kerala, India: Research Signpost; 2011. p. 93-110.

28. Silva EB, Dow SW. Development of Burkholderia mallei and pseudomallei vaccines. Front Cell Infect Microbiol. 2013;3:10

29. Scott AE, Laws TR, D'Elia RV, Stokes MG, Nandi T, Williamson ED, et al. Protection against experimental melioidosis following immunization with live Burkholderia thailandensis expressing a manno-heptose capsule. Clin Vaccine Immunol. 2013;20:1041-7.

30. Silva EB, Goodyear A, Sutherland MD, Podnecky NL, GonzalezJuarrero M, Schweizer HP, et al. Correlates of immune protection following cutaneous immunization with an attenuated Burkholderia pseudomallei vaccine. Infect Immun. 2013;81:4626-34. This is the first study that explores correlates of protection elicited against meliodosis based on subcutaneous route of vaccine administration.

31. Puangpetch A, Anderson R, Huang YY, Saengsot R, Sermswan RW, Wongratanacheewin S. Comparison of the protective effects of killed Burkholderia pseudomallei and $\mathrm{CpG}$ oligodeoxynucleotide against live challenge. Vaccine. 2014;32:5983-8.
32. Nieves W, Asakrah S, Qazi O, Brown KA, Kurtz J, Aucoin DP, et al. A naturally derived outer-membrane vesicle vaccine protects against lethal pulmonary Burkholderia pseudomallei infection. Vaccine. 2011;29:8381-9.

33. Nieves W, Petersen H, Judy BM, Blumentritt CA, Russell-Lodrigue $\mathrm{K}$, Roy CJ, et al. A Burkholderia pseudomallei outer membrane vesicle vaccine provides protection against lethal sepsis. Clin Vaccine Immunol. 2014;21:747-54.

34. Petersen H, Nieves W, Russell-Lodrigue K, Roy CJ, Morici LA. Evaluation of a outer membrane vesicle vaccine in nonhuman primates. Procedia Vaccinol. 2014;8:38-42. This study demonstrated that, in addition to OMV's ability to provide significant protection against respiratory meliodosis, it could also be safely administered to NHP's without any adverse effects.

35. Scott AE, Ngugi SA, Laws TR, Corser D, Lonsdale CL, D'Elia RV, et al. Protection against experimental melioidosis following immunisation with a lipopolysaccharide-protein conjugate. J Immunol Res. 2014;2014:392170.

36. Scott AE, Burtnick MN, Stokes MG, Whelan AO, Williamson ED, Atkins TP, et al. Burkholderia pseudomallei capsular polysaccharide conjugates provide protection against acute melioidosis. Infect Immun. 2014;82:3206-13.

37. Gregory AE, Judy BM, Qazi O, Blumentritt CA, Brown KA, Shaw $\mathrm{AM}$ et al. A gold nanoparticle-linked glycoconjugate vaccine against Burkholderia mallei. Nanomedicine. 2015;11:447-56.

38.• Torres AG, Gregory AE, Hatcher CL, Vinet-Oliphant H, Morici LA, Titball RW, et al. Protection of non-human primates against glanders with a gold nanoparticle glycoconjugate vaccine. Vaccine. 2015;33:688-92. This is the first attempt at $\boldsymbol{B}$. mallei vaccination in an NHP model and validates NHPs as a glanders model through $\mathrm{LD}_{50}$ establishment.

39. Naureen A, Saqib M, Muhammad G, Hussain MH, Asi MN Comparative evaluation of Rose Bengal plate agglutination test, mallein test, and some conventional serological tests for diagnosis of equine glanders. J Vet Diagn Invest. 2007;19:362-7.

40. Duval BD, Elrod MG, Gee JE, Chantratita N, Tandhavanant S, Limmathurotsakul D, et al. Evaluation of a latex agglutination assay for the identification of Burkholderia pseudomallei and Burkholderia mallei. Am J Trop Med Hyg. 2014;90:1043-6.

41. Janse I, Hamidjaja RA, Hendriks AC, van Rotterdam BJ. Multiplex qPCR for reliable detection and differentiation of Burkholderia mallei and Burkholderia pseudomallei. BMC Infect Dis. 2013;13: 86.

42. Hsu HL, Huang HH, Liang CC, Lin HC, Liu WT, Lin FP, et al. Suspension bead array of the single-stranded multiplex polymerase chain reaction amplicons for enhanced identification and quantification of multiple pathogens. Anal Chem. 2013;85:5562-8.

43. Singha H, Malik P, Goyal SK, Khurana SK, Mukhopadhyay C, Eshwara VK, et al. Optimization and validation of indirect ELISA using truncated TssB protein for the serodiagnosis of glanders amongst equines. Scientific World Journal. 2014;2014:469407.

44.• Houghton RL, Reed DE, Hubbard MA, Dillon MJ, Chen H, Currie BJ, et al. Development of a prototype lateral flow immunoassay (LFI) for the rapid diagnosis of melioidosis. PLoS Negl Trop Dis. 2014;8:e2727. Although still in its prototype stage, this assay's ease of use and ability to detect $B$. pseudomallei in primary specimens make it a promising rapid diagnosis tool.

45. Sorenson AE, Williams NL, Morris JL, Ketheesan N, Norton RE, Schaeffer PM. Improved diagnosis of melioidosis using a 2dimensional immunoarray. Diagn Microbiol Infect Dis. 2013;77: $209-15$. 